\title{
Curso de Administración de Redes utilizando antiguas técnicas y modernos medios
}

\section{Course of Network Administration using old techniques and modern means}

\section{Curso de Administración de Redes}

\author{
Manuel José Linares Alvaro ${ }^{(1)}$, MSc \\ Jorge Luis López Presmanes, PhD. ${ }^{2}$ \\ Ligia Vanessa Sánchez Parrales, Ing. ${ }^{3}$ \\ Marcos Tulio Zambrano Zambrano, PhD. ${ }^{4}$ \\ (1) Universidad de Granma, Cuba. E-mail: cheche@udg.co.cu \\ (2) Ministerio de Educación Superior, Cuba. E-mail: pres@reduniv.edu.cu \\ (3) Instituto Tecnológico Superior Portoviejo, Ecuador E-mail: ligia1980@live.com \\ (4) Universidad Laica Eloy Alfaro de Manabí, Ecuador. E-mail: marcostuliozambrano@ hotmail.com
}

Contacto: cheche@udg.co.cu

\section{Recibido: 18-02-2020 Aprobado: 11-03-2020}

\section{Resumen}

Las modalidades educativas basadas en cualquiera de las formas del e-learning, adquieren cada vez más relevancia en la sociedad actual, pues por una parte y desde el punto de vista económico, las mismas se encuentran al alcance de millones de personas en el planeta, y por otra, eliminan las barreras espacio temporales que cualquier tipo de curso o actividad docente implican; sin embargo, aún existen materias y cursos que son eminentemente prácticos, y que su correcto aprendizaje tradicionalmente implica prescencialidad, acompañada de las tradicionales actividades prácticas con equipos y dispositivos imprescindibles para completar su adecuado aprendizaje. La idea de dictar un curso completamente a distancia, de administración y servicios de redes, una materia con las características descritas con anterioridad, encaminado a profesionales del ramo de las diferentes universidades y centros adscritos al Ministerio de Educación Superior (MES) de Cuba, surge para dar respuesta ante la necesidad de mejorar el nivel de preparación de los especialistas nóveles que se dedican a la actividad descrita; y lo novedoso de esta experiencia radica en el empleo de una plataforma virtual que contiene redes y dispositivos IT (ruteadores, switchs, etc), de manera que cada estudiante del curso, pueda contar con sus propios dispositivos de red, condicionándose de manera idónea y con menos recursos económicos, la adquisición de las habilidades prácticas que esta ciencia exige. Se concluye destacando el elevado nivel de satisfacción, aprendizaje y habilidades adquiridas por los estudiantes, y se recomienda la transformación del curso en un diplomado a distancia, destinado a todo el que quiera laborar como administrador de una red en cualquiera de las entidades adscritas el MES.

Palabras clave: Educación a distancia, redes de computadoras, educación online, virtualización

\begin{abstract}
The educational modalities based on any of the forms of e-learning, acquire more and more relevance in today's society, because on the one hand and from the economic point of view, they are within reach of millions of people on the planet, and on the other, they eliminate the space - time barriers that any type of teaching course or activity implies; However, there are still subjects and courses that are eminently practical, and that their correct learning traditionally implies presence, accompanied by traditional practical activities with essential equipment and devices to complete their adequate learning. The idea of teaching a completely distance course, of administration and network services, a subject with the characteristics described above, aimed at professionals from the different universities and centers attached to the Ministry of Higher Education (MES) of Cuba, arises to respond to the need to improve the level of preparation of the specialist specialists who are engaged in the activity described; and the novelty of this experience lies in the use of a virtual platform that contains networks and IT devices (routers, switches, etc.), so that each student of the course can have their own network devices, conditioned in an appropriate way and With less economic resources, the acquisition of practical skills that this science demands. It is concluded by highlighting the high level of satisfaction, learning and skills acquired by students, and the transformation of the course into a distance diploma
\end{abstract}


course is recommended, aimed at anyone who wants to work as a network administrator in any of the entities attached to the MES.

Keywords: e-learning, computer networks, online education, virtualization

\section{Introducción.}

La educación a distancia no es un una modalidad educativa reciente y mucho menos, exclusiva de las Tecnologías de la Información y las Comunicaciones (TICs), pues de este modo de enseñanza - aprendizaje se tienen antiguas referencias: Baelo (2009), señala que la introducción del e-learning en la educación va más allá de la introducción de las TIC en los procesos educativos y tiene en los principios de interacción y continuidad desarrollados por Dewey sus principales valedores pedagógicos, mientras que Keegan (1993), alega que se conoce que en 1833 se registró en Suecia un curso de Contabilidad por correspondencia, y en Inglaterra, en 1843, Isaac Pitman crea la Phonografic Corresponding Society para la enseñanza de la taquigrafía a distancia.

El autor antes mencionado destaca que la enseñanza a distancia, ha atravesado por 3 generaciones hasta la actualidad: la primera, caracterizada por constituir básicamente un modelo cerrado, centrado en el material didáctico; en el que la evaluación buscaba "medir" los resultados del aprendizaje, verificando a través de las tareas realizadas y exámenes, la cantidad de conocimientos transmitidos que fueron efectivamente asimilados por el estudiante. Existía interactividad pero extremadamente retardada y en función de los medios. En este punto, resulta recomendable señalar que para garantizar el éxito deseado en cualquier proceso docente, es imprescindible la existencia de una interactividad constante, fluida, eficiente y de uso sencillo, tanto del tipo profesor - estudiante como estudiante estudiante o, incluso, profesor - profesor, debido, sobre todo, a que la enseñanza es una forma especial de comunicación, y una comunicación verdadera, real, y eficaz, requiere que la misma fluya en ambos sentidos.

Keegan (1993), plantea la existencia de una segunda generación en la modalidad de enseñanza a distancia, la cual llama también "analógica", en la que la mediación de la enseñanza y el aprendizaje continúa efectuándose por medio de material impreso, pero que comienzan a tomar características específicas diseñadas para la enseñanza a distancia. En esta generación, se introdujo la divulgación de la información a través de la radio, la televisión y las cintas de audio y video magnetofónicas, sin embargo, la interactividad seguía siendo una barrera que conducía a un bajo porciento de estudiantes que concluían con éxito completo sus estudios.

Ya la tercera generación, la cual puede denominarse "digital", puede describirse a grandes rasgos con la integración de las TICs, y con esto el uso de la Internet y otros canales de distribución digital, el desarrollo alcanzado por la web (surgimiento de la web 2.0 y luego la 3.0) (Baelo, 2009), y otros sistemas muy novedosos, como son el empleo de dispositivos móviles, sistemas interactivos de video conferencias, el e-learning 2.0, etc.

Puede notarse que una de las características distintivas es la unidireccionalidad o escasa bidireccionalidad del proceso de comunicación enseñanza en la primera y segunda generación, en comparación con la bidireccionalidad de la tercera generación.

En esta última o fase de desarrollo de la enseñanza a distancia surge un nuevo término, denominado elearning, éste se ha convertido en una de las palabras actualmente de moda en educación (al extremo, que algunos se han hecho a la idea que la educación a distancia ha surgido o es un fenómeno propio del elearning). En muy pocos años este vocablo ha pasado del vocabulario utilizado por una minoría de expertos en las aplicaciones de la tecnología en la enseñanza a ser empleado por múltiples instituciones, empresas y agentes educativos. (Ally, 2008)

Si se toma como referencia la raíz de la palabra, elearning se traduce como "aprendizaje electrónico", y como tal, en su concepto más amplio puede comprender cualquier actividad educativa que utilice medios electrónicos para realizar todo o parte del proceso formativo. (García, 2005)

Autores como Area (2007) y Cardona Román \& Sánchez (2011), son del criterio que el e-learning, también es conocido como teleformación, educación virtual, cursos online, enseñanza flexible, educación web, docencia en línea, etc; y se entiende como una modalidad de enseñanza-aprendizaje que consiste en el diseño, puesta en práctica y evaluación de un curso o plan formativo desarrollado a través de redes de ordenadores y puede definirse como una educación o formación ofrecida a individuos que están geográficamente dispersos o separados o que interactúan en tiempos diferidos del docente empleando los recursos informáticos y de telecomunicaciones. (Area \& Adell, 2009). Lo característico del e-learning es que el proceso formativo tiene lugar totalmente o en parte a través de una especie de aula o entorno virtual en el cual tiene lugar la interacción profesor-alumnos así como las actividades de los estudiantes con los materiales de aprendizaje. 
El e-learning se presenta actualmente, como una de las estrategias formativas que puede resolver muchos de los problemas educativos en la enseñanza moderna, los cuales se extienden desde el aislamiento geográfico del estudiante de los centros de aprendizaje hasta la necesidad de perfeccionamiento constante que exige la actual sociedad del conocimiento, sin obviar, las ventajas relacionadas con la economía personal, el tiempo que supone, o la motivación que implica el empleo de las TICs y aunque es verdad que sobre esta modalidad de formación se ha depositado un gran número de esperanzas, algunas de las cuales es cierto que se han visto confirmadas, tampoco estaría mal recordar que hay más de un $80 \%$ de fracaso en la gestión de cursos a distancia y más de un $60 \%$ de abandono de los mismos por parte de los estudiantes.

Sin embargo, aún las estadías, fases o generaciones en el desarrollo de la enseñanza a distancia más modernas, adolecen de un problema: existen materias o asignaturas que no se adaptan bien a este tipo de enseñanza, por requerir o necesitar del desarrollo de ciertas habilidades en los estudiantes, que requieren de recursos, dispositivos y equipos, generalmente costosos y en extremo escasos, que no están al alcance de los estudiantes o público en general, incluso, que no siempre existen en los centros de estudios.

Las asignaturas o materias relacionadas con el aprendizaje de la administración, manejo de redes de computadoras así como sus principales servicios, son materias que "encajan" perfectamente en la situación descrita con anterioridad: un normal aprendizaje de las mismas, requieren de la adquisición de habilidades prácticas en la implementación y diseño de los servicios y servidores que se suministran a los usuarios a través de las redes, el manejo de sofisticados dispositivos de red (dispositivos IT), como son los Routers, los Switchs o incluso, la implementación de servicios de redes en servidores o "hosts".

\section{Situación problémica}

En Cuba, todas las universidades y centros de educación superior, centros de investigación, Institutos, etc, se subordinan al Ministerio de Educación Superior (MES) y todos cuentan con sus respectivos centros de datos y nodos de comunicaciones, en los cuales se hallan los servidores, servicios más importantes de cada entidad. Por otra parte hace unos años y con el propósito de intercambiar información científico técnica, optimizar el uso de recursos, agilizar los procesos de gestión, reducir costes, mejorar las comunicaciones internas y hacer más eficiente el propósito final de la Educación Superior, se creó una red privada que interconecta de manera privada a todas las entidades del MES entre sí; a esta red, reconocida bajo el nombre de RedUniv, cada miembro debe aportar determinado número de servicios a la comunidad universitaria, y debe aclararse además que en cada centro de datos tanto del MES como de cualquier otra institución que se le subordine, existe un conjunto de especialistas, ingenieros y técnicos de alto nivel, encargados de administrar los servicios, mejorar los ya existentes, proponer o desarrollar nuevas facilidades y servicios o también, solucionar a los problemas que frecuente e invariablemente suelen ocurrir en las redes.

A nivel nacional, en el MES y sus entidades se ha venido presentando un problema durante los últimos años, el cual se hace aún más profundo si se parte del criterio de que formar un buen administrador de red, requiere al menos 10 años de práctica y experiencias, y consiste en la poca estabilidad laboral del personal encargado de administrar los diferentes centros de datos de las instituciones que lo forman, se puede afirmar que anualmente, especialistas de experiencia cesan sus funciones por trasladarse a otras empresas $\mathrm{u}$ otros empleos mejor remunerados, muchos consiguen contratos de trabajo en otros países, por ello, es necesario constantemente contratar especialistas nóveles que no tienen la experiencia idónea para este tipo de trabajo, el proceso se convierte en un ciclo cerrado, pues una vez que los nuevos especialistas logran acumular la experiencia necesaria, comienzan a buscar otros empleos, ya sean en el país, o en el extranjero, que aporten mejores honorarios, fenómeno completamente humano e inevitable, cuya solución no está en retener al experto contratado, sino, en buscar la manera de formar de manera eficiente y rápida a los nuevos técnicos.

A partir de la detección de esta situación, profesionales con más de 25 años de experiencia y trabajo en la administración de redes pertenecientes a la dirección nacional de RedUniv, y a la Universidad de Granma, tuvieron la idea de diseñar un curso de redes que se dictara con una frecuencia anual y un carácter obligatorio para todo aquellos que comenzaran a desempeñarse como administradores de sistemas en los centros de datos y nodos de cualquiera de las universidades o centros adscritos al MES, de manera que se pudiera lograr un nivel mínimo de habilidades, conocimientos y experiencia requeridas para trabajar en la administración de redes en cualquier entidad del MES.

Las primeras ediciones de este curso se considerarían como un extenso curso de posgrado, para luego transformarlo en un diplomado y finalmente convertirlo en una Maestría en Redes.

La puesta en práctica de esta idea, planteó una primera barrera: por una parte, la enorme dificultad 
que implica el trasladado más de 25 personas de todo el país hacia un determinado lugar o sitio en que se dictara el curso, y por otra, el alto contenido de trabajo que deben afrontar diariamente estos especialistas en sus diferentes puestos de trabajo en contraposición con la necesidad de exonerarlos de sus obligaciones, para posibilitarles participar a tiempo completo en el proyecto antes mencionado. Por ello, se determinó que el curso debía ser completamente a distancia, pero la solución a un problema, por lo general implica otros: el cual se ha descrito en detalle con anterioridad: tanto la enseñanza de la administración y manejo de redes de computadoras como la implementación de servicios específicos, la configuración de dispositivos de red, el diseño de sistemas de seguridad, etc, requieren de recursos y equipos que no están disponibles a los estudiantes en la totalidad de las universidades cubanas, máxime que los dispositivos existentes de esta clase en todos los centros del MES, se encuentran en producción, por lo que no es recomendable emplearlos en la adquisición de habilidades para su manejo. Partiendo de la situación problémica descrita, entonces se puede plantear que el problema existente, consistió en la necesidad de nivelar los conocimientos y habilidades de manera rápida y eficiente del personal novel dedicado a la administración de las redes, servicios y servidores en las diferentes instituciones del MES, utilizando para ello un curso completamente a distancia basado en el e-learning.

\section{Objetivo.}

Teniendo entonces en cuenta el problema, se puede señalar que el objetivo de la investigación expuesta, consistió en implementar un curso virtual que integrara, por una parte las herramientas de comunicación, materiales y recursos utilizando el Sistema de Manejo de Contenidos Moodle y por otra parte, una plataforma de virtualización que fuera capaz de complementar mediante actividades prácticas y virtuales, el logro de las habilidades esperadas.

\section{Materiales y métodos.}

La investigación contó con varias etapas, la primera consistió en el diagnóstico y confirmación del problema científico descrito, para ello se realizaron entrevistas a profesores de experiencia en la asignatura, así como encuestas a ingenieros nóveles que trabajan actualmente en algún centro de datos del MES. También fueron entrevistados estudiantes de la carrera que ya habían aprobado la asignatura. En esta fase también se comenzó con la elaboración del estado del arte.

\section{Elección del software y las plataformas.}

Elección del LMS: Como se ha mencionado antes, la plataforma de educación a distancia, empleada como aula virtual fue Moodle (Vidrio, Gómez, \& Zambrano, 2015) (Sánchez \& Vidal, 2014). Esto se debió tanto a razones prácticas como científicas, pues la misma ha demostrado ser estable, con un amplio respaldo bibliográfico, extensas experiencias en su empleo, y resultados que demuestran su efectividad, pero además, en la Universidad de Granma existe un aula virtual de posgrado basada en este tipo de sistema, (http://eddist.udg.co.cu), con un servicio estable, rápido, y brinda buenas y abundantes prestaciones y facilidades tanto a los profesores como los estudiantes por lo que resultaba el sitio idóneo para desarrollar la el curso, sus materiales y recursos.

\section{Elección de la plataforma anfitriona de los recursos virtuales.}

Se analizaron varias posibles aplicaciones para ser empleadas, sin embargo, se establecieron ciertas pautas para elegir la más indicada a las necesidades del curso:

- Debería tratarse de un sistema basado en software libre, con código abierto.

- El sistema a utilizar tendría que contar con un serio respaldo bibliográfico, científicamente fundamentado.

- Ciertas preferencias por el software diseñado con objetivos educativos y con un reconocido nivel de utilización a escala mundial.

- Debía ser capaz de simular dispositivos de redes de la manera más similar a la realidad posible, y que éstos fueran accesibles desde redes externas, de forma tal que tanto estudiantes como profesores, pudieran trabajar de forma remota en ellos, como si se tratara de equipos reales.

Después de analizar varios sistemas, se determinó que el GNS-3 cumplía con todas las expectativas definidas previamente, sin embargo, antes fue necesario adquirir un servidor con la potencia suficiente, como para soportar varias redes, equipos y dispositivos, de manera que a cada estudiante le correspondiera un conjunto de recursos; para ello, se estimaron cursos de unos 25 participantes. Partiendo de las premisas antes señaladas, se determinó que hacía falta un ordenador con el hardware necesario como para soportar, de manera virtual, por cada estudiante:

- Una pequeña red (prefijo 29)

- Dos servidores virtuales basados en el sistema operativo Linux. 
- Un router virtual, preferentemente fabricado por Cisco, Systems.

- Un Switch de capa III. (Gil et al., 2014; Neumann, 2015; Velieva, Korolkova, \& Kulyavov, 2014; Zhen-bang \& Wei-feng, 2016).

- Un nombre de dominio.

\section{Elección del hardware adicional necesario.}

Se valoró entonces una computadora fabricada como servidor profesional, con no menos de 64 Gigas de RAM, fuentes de poder redundantes, arreglo RAID por hardware, y unos 5 dicos de 500 gigas aproximadamente. Tras algunas gestiones en el mercado, se localizó la venta de servidores profesionales de fabricación China marca Ispur, modelo NF5270M4, con 24 procesadores y los requerimientos antes descritos. Evidentemente, la calidad de los mismos no iguala a los de los grandes fabricantes como Dell, Hewlett Packard, sin embargo, presentan una inmejorable relación calidad - precio, con magnificas prestaciones.

Desde el momento que se obtuvo el servidor profesional, se le instaló el sistema operativo Fedora 24 Server para 64 bits, y el simulador GNS3-1.5.3all-in-one por contener éste los simuladores Dinamyps y el Dinagen. Las máquinas virtuales se simularon con la versión para Linux de Virtual Box. También fue preciso asignar direcciones IP y diseñar la red de ordenadores virtual que sería empleada en el curso. Este servidor fue instalado en el centro de datos de RedUniv, ubicado en las oficinas centrales del MES.

\section{Diseño de la infraestructura virtual:}

Para ello, fue preciso realizar el diseño del direccionamiento: a toda la estructura virtual se le designó un bloque con prefijo 24, el cual se fue segmentando como se explica a continuación.

La figura 1, muestra la estructura virtual creada con ayuda de los simuladores, en la que puede apreciarse, que el diseño de la plataforma virtual respondió a una red jerarquizada, la cual incluyó un router primario, identificado como "R1" que funcionaría como único punto de entrada y salida al resto de la red y administrable solo por los profesores del curso, luego una red, con prefijo 27 (32 direcciones), formada por el enlace entre la interface interna del router primario, en conexión mediante un switch virtual con el resto de los routers, de los cuales, se entregaría uno a cada estudiante (R2, R3, etc). Cada uno de estos routers secundarios funcionaría como puerta de entrada y salida a las redes interiores de cada uno, éstas con prefijo 29 y administradas y gestionadas por cada estudiante.

Cada red interna, contó con un switch capa III que se desempeñó para interconectar el router, con los dos servidores existentes en cada una.

\section{Modelos de dispositivos virtuales empleados:}

Todos los routers utilizados, fueron dispositivos Cisco, modelo C-7200, en las estaciones de trabajo se utilizó el Sistema Operativo Linux con la distribución Ubuntu-14.04.5 LTS y en los switchs virtuales, se utilizó el modelo Cisco Catalyst 3500. Una vez que se tuvo toda la infraestructura para prácticas virtuales a distancia, fue necesario diseñar el curso, el cual se creó, como se ha mencionado, en la plataforma virtual de posgrado de la Universidad de Granma. En todo momento se tuvo en cuenta la premisa de que se trataba de un curso destinado a administradores de redes, es decir, a especialistas con poca experiencia, pero que sin embargo, si poseían conocimientos elementales como la instalación de un sistema operativo en un ordenador, la configuración básica de cualquier sistema, los direccionamientos y encaminamientos básicos de rutas en redes IP, entre otros, por ello, se obviaron algunos de estos contenidos. 


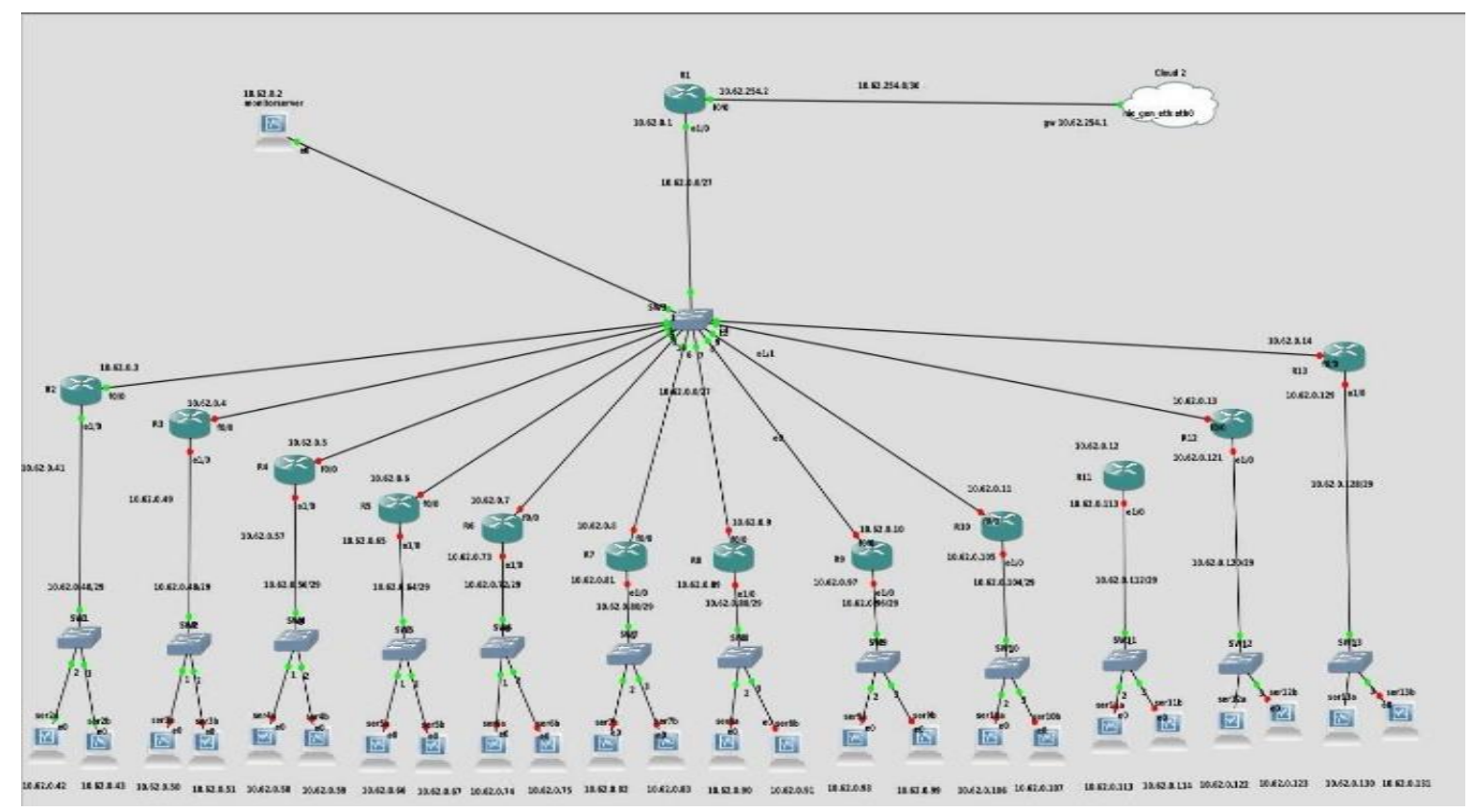

Figura 1. Diseño de la plataforma de virtualización empleada para el curso a distancia de Redes.

La primera edición del curso, contó con 4 temas:

- Una primera sección introductoria, donde se explicaban las características del curso, sus objetivos, fondos de tiempo, herramientas, aplicaciones de software y dispositivos virtuales puestos a disposición de cada estudiante, el modo y las credenciales para acceder a los dispositivos asignados, el sistema bibliográfico, los materiales, el sistema de evaluación y los sistemas de comunicación virtual de preferencia.

- Tema I. Servicios básicos. Contempla una serie de servicios comunes en todos los centros de datos de las redes actuales, por lo que el mismo se subdividió en varias unidades:

- Asignación de direcciones. Servidores DHCP.

- Resolución de nombres. Servidores DNS.

- Servidores de transferencia de archivos (FTP)

- Servicio Web. Servidor Apache

- Correo electrónico. Postfix y Dovecot

- Tema II. Encaminamientos avanzados y trabajo con routers y switchs fabricados por Cisco Systems.

- Tema III. Sistemas seguridad y de Gestión de la red. Este tema se subdividió en dos unidades:

- Sistemas de gestión

- Sistemas de seguridad (Seguridad)
- Tema IV. Gestión de información científico - Técnica. Este tema se incluyó con la finalidad de preparar a los estudiantes del curso, en la confección de la tesina, requisito considerado la evaluación final del mismo.

En el diseño del curso se empleó una estructura modular y siempre se trató de que la misma resultara homogénea para cada tema, por lo que puede señalarse que cada uno contó con las siguientes partes (figura 2):

- Foro para el debate de cuestiones relacionadas con el tema, así como para la aclaración de dudas, una vía de comunicación entre estudiantes y profesores.

- Bibliografía del tema.

- Conferencias o materiales de suministro de contenidos declarativos y teóricos.

- Generalmente, por cada conferencia, se crearon varios cuestionarios evaluativos para valorar la lectura y estudio de los materiales entregados a los estudiantes.

- También, por cada conferencia en el tema, se crearon "Tareas" que contenían las indicaciones para realizar trabajos independientes y la resolución de problemas empleando la plataforma virtual (Ej. Configuración y puesta a punto de un servidor DNS)

- Tras la finalización del segundo tema, se realizó una encuesta para conocer los 
niveles de satisfacción y de asimilación de los contenidos por parte de los estudiantes.

- Algunos temas contenían recursos multimedios con animaciones, videos, textos o cualquier material complementario de interés, los cuales formaron parte de la bibliografía del tema.

- Se especificaron detalladamente los objetivos de cada tema, cada material y cada actividad publicada.

\section{Sistema Bibliográfico.}

Para conformar el sistema bibliográfico del curso, se han empleado materiales digitales de todo tipo: libros en varios formatos, monografías escritas por los propios profesores del curso, materiales obtenidos en redes públicas, sitios webs, en fin, todo tipo de recurso que pudiera servir para complementar el proceso docente, sin embargo, se estableció que al menos el 70\% del sistema bibliográfico de cada tema, estuviera representado libros reconocidos, y que los mismos no tuvieran más de 4 años de antigüedad, así, son abundantes las obras y/o tratados de autores como O'Reilly, Tanembaum, CCNa, etc. Los contenidos declarativos, son distribuidos en forma de conferencias escritas, que contienen los aspectos principales de cada tema, unidad o sección de la materia tratada.
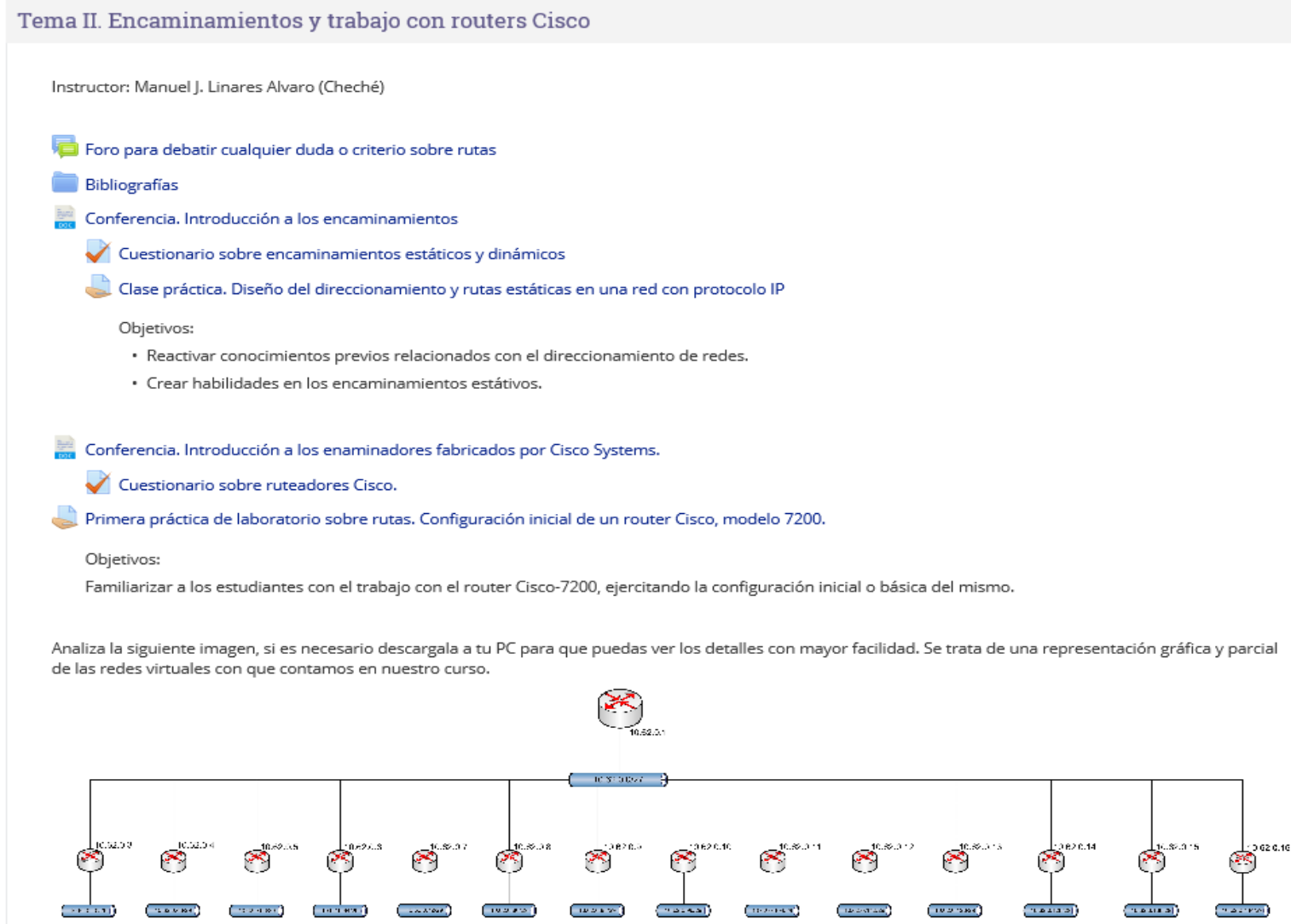

Figura 2. Vista parcial del Tema "Encaminamientos y trabajo con ruteadores Cisco" en la plataforma de enseñanza a distancia de la Universidad de Granma.

\section{Sistema de Evaluación.}

Se diseñó de manera que todas las actividades fueran evaluativas y partiendo del paradigma de considerar la evaluación como una ocasión más para aprender y una oportunidad adicional para mejorar la preparación de cada alumno. Por otra parte, cada conferencia, tiene sus propios cuestionarios para de este modo motivar al estudiante a que revise y analice los contenidos de la misma; cabe mencionar que alrededor del $80 \%$ de las actividades evaluativas, se basan en tareas que implican el envío de un informe final al profesor, para cuya solución, debe utilizar los recursos virtuales puestos a su disposición. Todos los profesores tienen accesos a los objetos virtuales de aprendizaje, para revisar, junto con el informe de la tarea, las soluciones propuestas y su funcionamiento. Como evaluación final, cada estudiante debe elaborar una tesina, que presentará a través de un foro de discusión creado para tal fin. 


\section{Sistema de contenidos.}

Los contenidos que se imparten en el curso, se han diseñado de manera tal, que se aborde, de manera práctica, la forma de implementar en los servicios básicos que debe suministrar cualquier centro de datos en una red avanzada. Por lo general, cada tema trata de abarcar uno de éstos. Se comienza por los servicios primarios, como son el direccionamiento IP, los encaminamientos, la asignación de direcciones, resolución de nombres, continuados por los servicios a los usuarios: directorios, correos, web, acceso a internet, etc; finalmente, se tratan servicios de seguridad y gestión de redes. El último tema se diseñó para preparar a cada estudiante en la confección de su tesina.

\section{Resultados.}

La primera edición de este curso, se impartió como algo experimental, sus veinte estudiantes procedían de centros de datos de diferentes redes e instituciones pertenecientes al MES, el mismo contó con un total de 160 horas clases, comenzó a impartirse a partir del 7 de noviembre de 2016 y se extendió hasta mayo de 2017. Este curso puede considerarse como un rotundo éxito si se valoran el hecho de que todos los estudiantes que lo iniciaron, concluyeron con buenos resultados y además, teniendo en cuenta e interpretando los resultados de las encuestas que se aplicaron durante el desarrollo del mismo.

Resumiendo los resultados de las encuestas, puede plantearse que el $100 \%$ de los estudiantes fue del criterio de que este curso sería de gran ayuda en el desempeño de sus actividades como administradores de sistemas, el $92 \%$ afirmó que se cumplieron sus expectativas por completo, mientras que un $8 \%$ fue del criterio que faltaron temas por tratar. Un $95 \%$ sostuvo que el curso tuvo una organización excelente y otro porciento similar expuso que cambiarán muchos aspectos en las redes que administran a partir de la recepción de este posgrado. Los estudiantes evalúan la preparación de los profesores como excelente, y la calidad de las plataformas empleadas como muy buenas.

Todos coinciden en sugerir la impartición periódica, al menos anual del curso, y que el mismo se transforme en un diplomado o maestría.

Las principales insatisfacciones de los estudiantes, estuvieron enfocadas a temas que no se abordaron, muchas veces por falta de recursos o falta de tiempo para su preparación.

Es importante señalar que en estos momentos se ha rediseñado todo el programa, de manera que las tres próximas ediciones previstas (actualmente se está dictando ya la tercera, que ha incluido una gran cantidad de nuevos contenidos), sean dictadas como un diplomado, de manera se logre un perfeccionamiento que permita convertirlo en un curso de maestría en redes, para 2021.

Es importante considerar que este trabajo representa un ejemplo de desarrollo de objetos virtuales de aprendizaje, combinados con la búsqueda de sistemas de aprendizajes interactivos, flexibles, accesibles con la conveniente integración de las TICs, lo cual parece ser uno de los retos presentes en los entornos virtuales de enseñanza hoy. (Trujillo, Hinojo, \& Aznar, 2016)

\section{Conclusiones.}

- Se diseñó un curso virtual de Administración de Redes, para los técnicos y especialistas que se desempeñan en cualquiera de las entidades pertenecientes al MES, en la modalidad e Learning, con prácticas de laboratorios y actividades virtuales.

- Tanto el Sistema de Manejo de Contenidos Moodle, como la plataforma de virtualización de prácticas de laboratorios, contribuyeron significativamente a favorecer el proceso de enseñanza-aprendizaje del curso y mejorar la motivación de los participantes.

- Se espera que este curso, de solución al problema de la inestabilidad en los recursos humanos que se desempeñan en los centros de datos de las instituciones pertenecientes a RedUniv y el MES, a través de la capacitación rápida de sus especialistas.

- En la nueva edición del curso, se están ensayando plataformas basadas en el empleo independiente de Dinamips, Routers basados en software y otras variantes que hagan un empleo más eficiente del hardware disponible.

\section{Referencias.}

Ally, M. (2008). The impact of technology on education (S. HIRTZ, HARPER, DG, \& MACKENZIE, S. Ed.).

Area, M. (2007). La docencia virtual en las universidades presenciales, Vol.1 y Vol.2. Revista Iberoamericana de Educación a Distancia, 10 y $11(2$ y 1$)$.

Area, M., \& Adell, J. (2009). e-Learning: Enseñar y Aprender en Espacios Virtuales: J. De Pablos (Coord): Tecnología Educativa. La formación del profesorado en la era de Internet. Aljibe, Málaga,.

Baelo, R. (2009). El e-Learning, una respuesta educativa a las demandas del siglo XXI. Pixel-Bit. Revista de Medios y Educación, $35,87-96$. 
Cardona-Román, D., \& Sánchez, Y. (2011). La Educación a Distancia y el E-Learning en la Sociedad de la Información: Una Revisión Conceptual. UIS Ingenierías, 10(1), 39 - 52.

García, F. (2005). Estado Actual de los sistemas de elearning.

http://www.redalyc.org/html/2010/2010210 55001/

Gil, P., García, G., Delgado, A., Medina, R., Calderón, A., \& Martí, P. (2014). Computer networks virtualization with GNS3: Evaluating a solution to optimize resources and achieve a distance learning IEEEE Splore. Digital Library. http://ieeexplore.ieee.org/abstract/documen t/7044343/

Keegan, D. (1993). Theoretical principles of distance education (pp. 9-21): RoutLedge.

Neumann, J. (2015). The Book of GNS3: Build Virtual Network Labs Using Cisco, Juniper, and More

Sánchez, M., \& Vidal, O. (2014). Aplicación de la Plataforma Moodle como herramienta didáctica para el desarrollo de competencias en estudiantes de Ingeniería. Revista Iberoamericana para la Investigación y el Desarrollo Educativo, Enero - Junio(12).

Trujillo, j., Hinojo, F., \& Aznar, I. (2016). Propuestas de trabajo innovadoras y colaborativas elearning 2.0 como demanda de la sociedad del conocimiento. Estudios sobre Educación, 20.

Velieva, T., Korolkova, A., \& Kulyavov, D. (2014). Designing installations for verification of the model of active queue management discipline RED in the GNS3 http://ieeexplore.ieee.org/abstract/documen t/7002164/

Vidrio, P., Gómez, M., \& Zambrano, D. (2015). Valoración didáctica del uso de Moodle en la educación media superior. Apertura. Revista de innovación educativa. Universidad de Guadalajara, 7 (1).
Zhen-bang, R., \& Wei-feng, G. (2016). Experimental teaching research of computer network engineering based GNS3 virtual laboratory. School of Computer Science,Wuyi University.

http://en.cnki.com.cn/Article_en/CJFDTOT AL-YSKT201503028.htm 